\title{
Representing Continuous Change in the Abductive Event Calculus
}

\author{
Kristof Van Belleghem, Marc Denecker, Danny De Schreye \\ Department of Computer Science, K.U.Leuven, \\ Celestijnenlaan 200A, B-3001 Heverlee, Belgium. \\ \{kristof, marcd, dannyd\}@cs.kuleuven.ac.be
}

\begin{abstract}
In this paper we extend the Abductive Event Calculus, a variant and extension of the Event Calculus of Kowalski and Sergot, with a representation for continuous change. We assume the change is not exactly known and use constraints to represent the available knowledge. The resulting logic program can be executed by the SLDNFA abductive procedure of Denecker and De Schreye. We show how our representation of continuous change combines with solutions to other problems in temporal reasoning, like indeterministic effects of actions, and how it can be used not only for temporal projection, but also to solve planning and diagnosis problems or any combination thereof.
\end{abstract}

\section{Introduction}

The Event Calculus (see [12]) is one of the many formalisms used for representing a changing world. The basic concepts are events and properties, events initiating and terminating periods of time during which properties hold.

Several modified versions of the Event Calculus have been used, for example in [19], [9] and [15], mostly to simplify the ontology and to eliminate problems occuring because of bidirectional persistence of properties (forward as well as backward in time). Extensions were introduced to improve the expressive power in several ways. One of the most important of these was the introduction of abduction, for example in [8], [15] and [5], which made it possible to use the Event Calculus for planning and for diagnosis ([7]) as well as for temporal projection.

In the original Event Calculus, as in most other versions, all change is supposed to be discrete. Recently there have been proposals ([19], [16]) to incorporate continuous change in the Event Calculus, describing changing quantities as exactly known functions of time. In this paper we propose an extension of the Abductive Event Calculus that does not require such complete knowledge about the change, and which is closer to the qualitative reasoning point of view. One other qualitative approach to representating continuous change in the Event Calculus is described in [20], but as the author points out, that work was never really completed.

We will apply our formalism to a number of examples and show how it can be used to solve projection, planning and diagnosis problems and problems in which indeterminism occurs. 


\section{The Event Calculus}

In the Event Calculus, information is represented in Horn clauses augmented with negation as failure. The following axioms define a simplified version of the Event Calculus, which we use as a basis for introducing our extension:

$$
\begin{aligned}
\text { holds_at }(P, T) \leftarrow & \text { happens }(E), E<<T, \text { initiates }(E, P), \\
& \text { not clipped }(E, P, T) . \\
\operatorname{clipped}(E, P, T) \leftarrow & \text { happens }(C), \operatorname{in}(C, E, T), \\
& \text { terminates }(C, P) . \\
\operatorname{in}(C, E, T) \leftarrow & E<<C, C<<T .
\end{aligned}
$$

happens $(E)$ holds if the event $E$ occurs. We allow only one event to occur at any one time point, which makes it possible to represent events directly by their time of occurrence. This does not limit the expressive power of the formalism, since it is possible for more than one action to take place during one event. We consider events to be just special time points, on which for example actions may occur. A strict chronological order $<<$ is defined on all time points, including events.

The actions associated with an event determine which properties are initiated or terminated by it. This is formulated through domain dependent rules which are represented in the form

$$
\begin{aligned}
& \text { initiates }(E, P) \\
& \text { terminates }(E, P) \leftarrow \operatorname{act}(E, A), \operatorname{Precond}(A, P) . \\
& \leftarrow \operatorname{act}(E, B), \operatorname{Precond}(B, P) .
\end{aligned}
$$

where the optional Precond $(A, P)$ consists of a number of preconditions for the action, mostly in the form of holds_at and not holds_at expressions.

To represent problems in which an initial state is given, a start event is introduced. This event initiates all properties that are true in the initial state:

$$
\begin{aligned}
& \text { happens }(\text { start }) . \\
& \text { initiates }(\text { start }, P) \quad \leftarrow \quad \text { initially }(P) .
\end{aligned}
$$

No events are allowed to occur before start.

\section{SLDNFA in the Event Calculus}

The Event Calculus can be used as a logic program under Clark completion semantics ([2]) to solve temporal projection (prediction) problems. This can be done deductively using the well-known SLDNF procedure. However, Clark completion semantics assumes complete knowledge about the problem domain. Any form of incomplete knowledge - like actions with indeterministic effects - can not be dealt with. Furthermore, postdiction (diagnosis) and planning problems can not be solved using deduction in this form of the Event Calculus.

These problems can be overcome in the following way: on the representational level, we use the Console completion semantics for abductive logic programs of [3], augmented with general first order logic constraints. This semantics allows for the use of undefined predicates: predicates with an unknown truth value. Thus, incomplete knowledge can be represented. 
On the level of problem solving, we will use abduction as well as deduction, which requires an abductive proof procedure: given a set of logic formulas $F$ (facts and rules about the problem domain) and a number of conclusions $G$, an abductive procedure attempts to find a set of additional facts $\Delta$ such that

- $F+\Delta$ is consistent.

- $F+\Delta \models G$.

- $\Delta$ is minimal: no subset of $\Delta$ exists that satisfies the first two conditions.

The minimality condition is not always added. The facts allowed in $\Delta$ will be constrained by the user to obtain useful results.

Abduction can be used to deal with indeterministic effects and to solve diagnosis and planning problems, by constraining the facts in $\Delta$ in the appropriate way. In general the predicates allowed in $\Delta$ are those we have incomplete knowledge about, in other words the undefined predicates. In the sequel we refer to these as the abducible predicates. For example, in planning problems we have incomplete knowledge about the actions occurring in the plan and the time relations that connect them, so happens, act and $<<$ will be abducible. We try to find the sequence of actions necessary to prove the goal, which is the desired end state. In a similar way postdiction problems and indeterminism can be modeled.

For our experiments we have used an implementation of the SLDNFA (SLD resolution with Negation as Failure and Abduction) procedure described in [6] and [7]. This procedure allows for a better treatment of abducible atoms containing variables compared to other procedures. A proof of the soundness and completeness of the procedure with respect to Console completion semantics can be found in the aforementioned papers.

An important feature of this procedure is that it keeps track of both a list of goals for which a successful derivation is needed, and a list of goals for which finite failure must be proven. Therefore it is quite easy to handle constraints of the form

$$
\text { false } \leftarrow A_{1}, \ldots A_{n} .
$$

by adding $\leftarrow A_{1}, \ldots A_{n}$. to the latter list of goals. The use of this kind of constraints proved very helpful in many situations, not in the least in our proposal for modeling continuous change. In general we can transform any logic formula into one or more rules of this form ([14]), thereby extending the expressiveness of the formalism considerably. Given the program $F$ including the set of constraints, and the goal " $\neg$ false \& $G$ ", the SLDNFA procedure finds a $\Delta$ such that

$$
\operatorname{Comp}(F+\Delta) \models \neg \text { false \& } G
$$

where $\operatorname{Comp}(P)$ is the Clark completion of $P$. Note that the atom false should not be interpreted as simply $\neg$ true: it actually means "a constraint is violated", and the goal $\neg$ false is then used to ensure satisfaction of all constraints. 


\section{Continuous Change}

Like most other formalisms developed for temporal reasoning, the Event Calculus originally did not take the possibility of continuous change into account. Every property takes on a number of discrete values (most often just booleans), and changes of these values occur at certain isolated time points. In many cases such an approach is sufficient: many properties have a boolean value or can only take on a limited number of discrete values. It is even possible to model a continuously changing value at a high level of abstraction using discrete values. One can for example use the properties on $(X, A)$, on $(X, B)$ and between $(X, A, B)$ to model the location of $X$, even though that location is in fact a continuous variable.

But for some problems this level of abstraction is too high: it can be used to model the continuous value, but it is insufficient if we want to model the change itself. An example where we need to model continuous change is the problem of a tank that can be filled with water, as introduced in [19]. This is a simple problem, yet it shows many of the complications that arise when continuous change occurs. The most important of these is the problem of autotermination, also introduced in [19]. It is possible that the change of a property over time gets terminated by itself, without the occurrence of any external event. This happens for example when the rising water in a tank reaches its rim. At that moment, the change in water level causes the event of its own termination.

Other complications of this form can occur if the water triggers events when it reaches certain levels, like the ringing of a warning bell. In general, a changing property can cause a number of other events to happen at different time points.

The water tank problem can be extended in several ways, and we can use it to demonstrate a number of different types of problem solving. For example, it is possible to introduce a number of taps and plugs (open taps fill the tank, open plugs empty it) that can be opened or closed simultaneously. We can introduce several warning bells, as discussed earlier, and allow for the possibility that some of these bells are broken (indeterministic aspect). We can then open a tap without closing it later, and ask why no bell has rung (diagnosis : the bell must be broken). We can ask whether the opening of the tap will result in bells ringing (two solutions: no if they are broken, yes otherwise). We can open a tap and ask to avoid the ringing of any bells, even though they are not broken (planning: the tap must be closed, or a plug opened, or maybe the bell turned off).

When looking for a representation of continuous change, two approaches can be distinguished. The first one assumes complete knowledge about the change: the changing quantity is exactly known as a function of time, or can be calculated from other data (like the flow through a certain tap). This approach is taken by most authors, for example in [17], [19] and [16].

But in the case of continuous change - even more than in other cases it seems necessary to allow for incomplete knowledge. For example, while it is easy to check whether a turkey is dead or alive, determining the water level in a filling tank as an exact function of time is non-trivial. Most probably the 
only real knowledge available is that the level is rising. We will show that even such very limited knowledge contains important information that can be used in several types of problem solving.

\section{Representation of continuous change in the Ab- ductive Event Calculus}

We propose a representation of continuous change that only requires qualitative knowledge. For example, in the case of a filling water tank such knowledge might be that the water level is rising, that it is rising in a continuous way, that autotermination will occur when the water reaches the rim, etc. We want this solution to fit in with the general framework of the Abductive Event Calculus, so that it can be combined with discrete changes, indeterminism, and planning and diagnosis problems.

We present our proposal in two stages: in a first step we allow only one influence to exist on a changing variable at the same instant in time. In the second step we extend this solution with simultaneous influences. We illustrate the formalism by modeling a filling water tank and using the representation for problem solving.

\subsection{Solution without simultaneous influences}

In our first step we introduce two new predicates. cont_change $(P, S o r t, T)$ holds if at time point $T$ the property $P$ is subject to a continuous change of sort $S$ ort. Sort is a parameter used to distinguish different kinds of change, where each kind has certain unique properties. How many and which kinds of change are to be distinguished, depends on the amount of available knowledge as well as on the relevance of the observed differences between two kinds. A lot of work on this topic of making useful and adequate abstractions exists in the qualitative physics community (see for example [10] and [13]). One simple and obvious abstraction, which is often used in qualitative physics, is the distinction between positive and negative change. We will use this distinction in our examples.

state_in_change $(P, T)$ is a predicate describing the value of $P$ as a function of time during periods of change. We add the following axiom to the Event Calculus to express this:

$$
\begin{aligned}
\operatorname{holds\_ at}(P, T) \leftarrow \quad & \text { cont_change }(P, S o r t, T), \\
& \text { state_in_change }(P, T) .
\end{aligned}
$$

So, if $P$ is changing, its value is defined by state_in_change. The following axioms describe when a continuous change is in effect.

$$
\begin{aligned}
\text { cont_change }(P, S o r t, T) \leftarrow & \text { happens }(E), E<<T, \\
& \text { init_change }(E, P, S \text { ort }), \\
& \text { not change_clipped }(E, P, T, \text { Sort }) . \\
\text { change_clipped }(E, P, T, S o r t) \leftarrow & \text { happens }(C), \text { in }(C, E, T), \\
& \text { term_change }(C, P, S \text { ort }) .
\end{aligned}
$$


where init_change, term_change and change_clipped correspond to initiates, terminates and clipped for discrete properties.

During periods of change, the state_in_change function determines the value of properties. This approach is similar to the definition of trajectories in [19], but where trajectories are defined as exactly known functions, we leave state_in_change undefined (abducible). Instead we define constraints on the state_in_change function to represent the available knowledge, for example monotonicity or continuity of the change. These constraints will be described later on. We will show that they provide the expressiveness needed to handle all aforementioned applications.

The original axioms of the Event Calculus still apply, and describe the state of properties while they are not undergoing any change. To ensure correct interaction between periods of change and periods of rest, we add the following axioms:

$$
\begin{array}{ll}
\text { terminates }(E, P) & \leftarrow \text { init_change }(E, P, \text { Sort }) . \\
\text { initiates }(E, P) & \leftarrow \text { holds_at }(P, E), \text { term_change }(E, P, S o r t) .
\end{array}
$$

meaning that the start of a change terminates a period of rest, while termination of the change initiates a new period of rest. In particular, the case in which a change has terminated and no new change initiated, is now properly dealt with by the holds_at rule for discrete change.

We will illustrate the use of this formalism by describing a filling water tank. The constraints we define are problem specific, since they depend on the actual knowledge available about the change. However, most of them represent quite common properties, like continuity of change, and can be generalized or adapted to other problem descriptions.

The tank contains a tap and a plug. An open tap results in rising water level, an open plug in dropping level. The tap and the plug can not be open simultaneously, since in this first step we do not allow multiple influences on one property at the same moment:

$$
\begin{array}{ll}
\text { init_change }(E, \text { level }(L),+) & \leftarrow \text { act }(E, \text { open_tap }) . \\
\text { term_change }(E, \text { level }(L),+) & \leftarrow \text { act }(E, \text { close_tap }) . \\
\text { init_change }(E, \text { level }(L),-) & \leftarrow \text { act }(E, \text { open_plug }) . \\
\text { term_change }(E, \text { level }(L),-) & \leftarrow \text { act }(E, \text { close_plug }) . \\
\text { false } \leftarrow \quad \text { cont_change }(E, P,-), \text { cont_change }(E, P,+) .
\end{array}
$$

In this example we choose to distinguish only two kinds of continuous change: rising and dropping water level, denoted by sorts + and -. As indicated earlier, this is a very simple abstraction, but we will show its usefulness. The changing property we consider is $\operatorname{level}(L)$, the water level in the tank. We know the following:

- The water level is rising (dropping) monotonically.

- At any instant in time, there can be only one level.

- The change is continuous (if the water reaches two different levels during one period of change, then it will also reach all levels between them). 
- If the tap is opened, and nothing happens that stops the rising of the water, then the water will eventually reach the rim of the tank (we assume the water will not do strange things like rise asymptotically to the rim). Similarly the tank will eventually become empty if the plug is open.

- When the rising (dropping) water reaches the rim (bottom) of the tank, the change is automatically terminated.

This information is expressed in a number of constraints, written in the form required by SLDNFA. In order to be able to write these constraints, we first specify when two time points belong to a same period of change:

$$
\begin{aligned}
\text { same_change }\left(P, S o r t, T_{1}, T_{2}\right) \leftarrow & \text { happens }(C), C \leq T_{1}, T_{1}<T_{2}, \\
& \text { init_change }(C, P, S o r t), \\
& \text { not change_clipped }(C, P, T 2, \text { Sort }) .
\end{aligned}
$$

where $\leq$ is defined in the usual way.

Next, we define a linear order on the set of water levels. A special constraint module has been added to our SLDNFA-implementation to efficiently keep track of any number of such ordered sets, ensuring they have a strict linear order defined on them. The formula is $a(X, O)$ is used to indicate that $X$ belongs to set $O$. The linear order is described by the predicate smaller $/ 3:$ smaller $(O, X, Y)$ holds if $X$ is smaller than $Y$ with respect to the order on $O$. From a declarative point of view, smaller $/ 3$ is an undefined predicate satisfying antireflexivity, antisymmetry, transitivity and linearity constraints.

Moreover, if a scenario asserts isa(bottom $(O), O)$ and/or isa(top $(O), O)$, these are the extrema of $O$. Otherwise no extrema exist. The set $O$ does not need to be completely determined: it is possible to make isa abducible (satisfying certain constraints of course). For example, this allows us to consider only the relevant levels in a tank, with the possibility to introduce more levels if (and only if) needed.

In fact, the order $<<$ on events is a variant of this general linear order, with start being the minimum of the set and no maximum defined. Similarly happens is a special case of isa.

We refer to the set of water levels as l_type, and add the constraint

$$
\text { false } \leftarrow \text { holds_at }(\text { level }(X), T) \text {, not isa }\left(X, l_{-} \text {type }\right) \text {. }
$$

Now we can formulate our constraints on changes. The constraints are written in terms of holds_at, but actually - through the rules for holds_at in terms of state_in_change - constrain this undefined predicate. The first of the constraints, concerning monotonicity, ensures that for every two time points during the same period of positive change, the level on the later time point is greater 
than the level on the earlier one. The opposite holds for negative change.

$$
\begin{aligned}
& \text { false } \leftarrow \text { same_change }\left(\text { level }(L),+, T_{1}, T_{2}\right), \text { holds_at }\left(\text { level }(X), T_{1}\right) \text {, } \\
& \text { holds_at(level } \left.\left.(Y), T_{2}\right), T_{1}<<T_{2} \text {, smaller (l_type, } Y, X\right) \text {. } \\
& \text { false } \leftarrow \text { same_change }\left(\text { level }(L),+, T_{1}, T_{2}\right) \text {, holds_at }\left(\text { level }(X), T_{1}\right) \text {, } \\
& \text { holds_at(level } \left.(X), T_{2}\right), T_{1}<<T_{2} \text {, not } X=\text { top (l_type). } \\
& \text { false } \left.\leftarrow \text { same_change }\left(\text { level }(L),-, T_{1}, T_{2}\right) \text {, holds_at (level }(X), T_{1}\right) \text {, } \\
& \text { holds_at(level } \left.\left.(Y), T_{2}\right), T_{1}<<T_{2} \text {, smaller (ltype, } X, Y\right) \text {. } \\
& \text { false } \leftarrow \text { same_change }\left(\text { level }(L),-, T_{1}, T_{2}\right) \text {, holds_at }\left(\text { level }(X), T_{1}\right) \text {, } \\
& \text { holds_at(level } \left.(X), T_{2}\right), T_{1}<<T_{2} \text {, not } X=\text { bottom(l_type). }
\end{aligned}
$$

We allow for the level to remain constant once the water reaches the rim. This, together with the restriction that the rim is the maximum existing level, captures the meaning of autotermination: when the tank is full, the level stops rising. We choose this representation rather than introducing a terminating event caused by the change like Shanahan does. Such autoterminating event would not distinguish the case in which the tap is closed just when the tank is full from the case in which the tank overflows. However, there are differences, like the floor getting wet. Moreover, in our next step we will allow for multiple simultaneous influences on a changing variable. In that case, the introduction of an autoterminating event leads to erroneous conclusions, as we will discuss later. In our representation the tank being full does not terminate the period of change, even though the level remains constant.

The other constraints look like this:

- no two levels at the same instant:

false $\leftarrow$ holds_at $\left(\right.$ level $\left.(X), T_{1}\right)$, holds_at(level $\left.(Y), T_{1}\right)$, not $X=Y$.

- continuity:

$$
\begin{aligned}
& \text { false } \leftarrow \text { same_change }\left(\text { level }(L), \text { Sort }, T_{1}, T_{2}\right), T_{1}<<T_{2} \text {, } \\
& \text { holds_at (level } \left.\left.(X), T_{1}\right) \text {, holds_at(level }(Y), T 2\right) \text {, isa }(Z, l \text { type }) \text {, } \\
& \text { between (l_type, } \left.Z, X, Y) \text {, not reach_between(l_type, } Z, T_{1}, T_{2}\right) \text {. } \\
& \text { reach_between }\left(l_{-} \text {type, } Z, T_{1}, T_{2}\right) \leftarrow \text { happens }\left(T_{3}\right), T_{1}<<T_{3}, T_{3}<<T_{2} \text {, } \\
& \text { holds_at }\left(\text { level }(Z), T_{3}\right) \text {. } \\
& \text { between }(O, Z, X, Y) \leftarrow \text { smaller }(O, X, Z) \text {, smaller }(O, Z, Y) \text {. } \\
& \text { between }(O, Z, X, Y) \leftarrow \text { smaller }(O, Y, Z) \text {, smaller }(O, Z, X) \text {. } \\
& \text { - water eventually reaches the rim: } \\
& \text { false } \leftarrow \text { happens }(E) \text {, init_change }(E, \text { level }(X),+) \text {, } \\
& \text { not change_clipped_after }(E \text {, level }(X),+) \text {, } \\
& \text { isa(top(l_type), l_type), not reach_after }\left(E, \operatorname{top}\left(l_{-} t y p e\right)\right) \text {. } \\
& \text { false } \leftarrow \text { happens }(E) \text {, init_change }(E \text {, level }(X),-) \text {, } \\
& \text { not change_clipped_after }(E, \text { level }(X),-) \text {, } \\
& \text { isa(bottom(l_type), l_type), not reach_after }(E, \text { bottom(l_type)). } \\
& \text { reach_after }(E, L) \leftarrow \text { happens }\left(E_{2}\right), E<<E_{2} \text {, holds_at }\left(\text { level }(L), E_{2}\right) \text {. } \\
& \text { change_clipped_after }(E, P, S \text { ort }) \text { thappens }\left(E_{2}\right), E<<E_{2} \text {, } \\
& \text { term_change }\left(E_{2}, P, S o r t\right) \text {. }
\end{aligned}
$$




\subsection{Applications}

This version of our proposal can already handle a variety of problems. First we study a simple scenario in the water tank world. We define three levels: the bottom of the tank, the top, and a level halfway. The tank is initially empty, and then a tap is opened.

Although in practice we can and most often will make happens and $<<$ abducible, this usually implies that infinitely many solutions to a given query exist. Using an iterative deepening control in the SLDNFA implementation, solutions with a minimal number of abduced events can be generated first. Since we do not want to elaborate on this implementation in the current paper, we instead assume that the necessary number of events is added to the scenario. We emphasize, however, that this is not a limitation of the approach. In this scenario, we add two additional events.

$$
\begin{aligned}
& \text { happens(start). happens }\left(e_{1}\right) \text {. happens }\left(e_{2}\right) \text {. happens }\left(e_{3}\right) \text {. } \\
& \text { start }<<e_{1} . \quad e_{1}<<e_{2} . \quad e_{2}<<e_{3} . \\
& \text { isa(bottom(l_type), l_type). } \quad \text { isa(half,l_type). } \quad \text { isa(top(l_type), l_type). } \\
& \text { initially(level(bottom(ltype))). act }\left(e_{1}, \text { open_tap }\right) \text {. }
\end{aligned}
$$

We want to know if, and under which conditions, this scenario is possible. So we try to solve the query " $\leftarrow$ not false". We obtain the following abduced facts:

$$
\begin{aligned}
& \text { state_in_change }\left(\text { level }(\text { half }), e_{2}\right) . \\
& \text { state_in_change }\left(\text { level }(\text { top }(l+t y p e)), e_{3}\right) .
\end{aligned}
$$

and a couple of facts ordering the l_type set. This order is trivial, since there is only one level apart from the minimum and maximum of the set.

This solution is indeed correct: because the tap is never closed, the water reaches the top. This can happen no later than at time $e_{3}$, since $e_{3}$ is the last time point we defined. Now, since the water reaches the top, we know it also reaches all levels between bottom and top. So, the water must reach the level halfway sometime between $e_{1}$ and $e_{3}$, which can only be at $e_{2}$, the only other event we provided. Of course, if other events existed between $e_{1}$ and $e_{3}$, and especially if happens were abducible, there would be other solutions as well.

In a second example we add a bell that rings when the water reaches the level halfway. This shows how we can handle events that are caused by the change. At the same time we add an indeterministic aspect, by making it possible for the bell to be broken. We do not know whether the bell is broken or not, so we declare broken_bell to be an abducible predicate, as in [7]. We only have to add the rule

$$
\text { initiates }(E, \text { ring_bell }) \leftarrow \text { holds_at(level(half }), E) \text {, not broken_bell. }
$$

With the bell and the indeterminism added, we can demonstrate how diagnosis problems are handled. To diagnose why the bell is not ringing at $e_{3}$, we use the query

$$
\left.\leftarrow \text { not false, not holds_at (ringbell, } e_{3}\right) \text {. }
$$


The additional abduction of broken_bell is made, since the level halfway has to be reached at $e_{2}$. A solution without broken_bell does not exist.

Postdiction problems, where facts about an earlier time point are derived given information about a later one, are a special form of diagnosis problem where initially is the abducible predicate.

Finally we can use this representation with the SLDNFA procedure for planning in the context of continuous change. As an example, we generate a plan of actions that explains the fact that, after opening a tap, the bell does not ring even though it is not broken. We have the general rules

$$
\begin{array}{ll}
\text { init_change }(E, \text { level }(X),+) & \leftarrow \text { act }(E, \text { open_tap }) . \\
\text { term_change }(E, \text { level }(X),+) & \leftarrow \text { act }(E, \text { close_tap }) . \\
\text { initiates }(E, \text { ring_bell }) & \leftarrow \text { holds_at(level }(h a l f), E), \text { not broken_bell } .
\end{array}
$$

The definitions of happens, isa, initially and $<<$ are identical to those in our first application, and state_in_change, smaller, act and broken_bell are abducible. If we want to solve

$\leftarrow$ not false, act( $e_{1}$, open_tap $)$, not holds_at(ringbell, $\left.e_{3}\right)$, not broken_bell .

we find one solution with abduced facts act $\left(e_{1}\right.$, open_tap $)$ and $a c t\left(e_{2}\right.$, close_tap $)$, and a second one with the close_tap action on $e_{3}$ instead of $e_{2}$. Because the tap is now closed at a certain point in time, the water is no longer guaranteed to reach the rim, or even the level halfway. The level at time $e_{3}$ will be somewhere between bottom(l_type) and half.

\subsection{Extension for multiple influences}

If we want to allow for multiple simultaneous influences to exist on the same changing variable, we need to extend our proposal. Where the notion of influence, or in fact the distinction between change and influence was unimportant in our previous version, it is now of vital importance. We introduce a new predicate influence/4. influence $(I, P$, Sort,$T)$ holds if at time point $T, P$ is subject to the influence $I$ of sort $S$ ort.

Changes are now defined in terms of the existing influences, while the effect of actions is the initiation and/or termination of these influences. The predicates init_change, term_change and change_clipped will be eliminated and replaced by a set of new predicates init_influ, term_influ, influ_clipped, influ_started and influenced. The following new definitions apply:

$$
\begin{aligned}
& \text { influence }(I, P, S, T) \quad \leftarrow \text { happens }(E), E<<T \text {, init_influ }(E, I, P, S) \text {, } \\
& \text { not influ_clipped }(I, E, P, T) \text {. } \\
& \text { influ_clipped }(I, E, P, T) \leftarrow \text { happens }(C), \text { in }(C, E, T), \text { term_influ }(C, I, P, S) \text {. } \\
& \text { influ_started }(I, E, P, T) \leftarrow \text { happens }(C), i n(C, E, T), \text { init_influ }(C, I, P, S) \text {. } \\
& \text { influenced }\left(T_{1}, P, T_{2}\right) \leftarrow \text { influ_clipped }\left(I, T_{1}, P, T_{2}\right) \text {. } \\
& \text { influenced }\left(T_{1}, P, T_{2}\right) \quad \leftarrow \quad \text { influ_started }\left(I, T_{1}, P, T_{2}\right) \text {. }
\end{aligned}
$$

We redefine cont_change in terms of influences, and leave our frame axioms and constraints unchanged. We choose to distinguish two kinds of influence: 
positive and negative. This leads to three kinds of change: if all influences on a variable are positive, the change is positive $(+)$. If all influences are negative, the change is negative $(-)$. If there are both positive and negative influences, the change is continuous but with unknown direction (?). This is expressed in the following rules:

$$
\begin{aligned}
& \text { cont_change }(P,+, T) \leftarrow \text { influence }(I, P,+, T), \text { not any_influ }(P,-, T) . \\
& \text { cont_change }(P,-, T) \leftarrow \text { influence }(I, P,-, T), \text { not any_influ }(P,+, T) . \\
& \text { cont_change }(P, ?, T) \leftarrow \text { influence }(I, P,+, T), \text { influence }(J, P,-, T) . \\
& \text { any_influ }(P, S o r t, T) \leftarrow \text { influence }(J, P, S \text { ort }, T) .
\end{aligned}
$$

where the last rule is introduced to avoid floundering.

As in the first proposal, the types of change and influence are chosen because of their generality. They can be modified if the problem domain requires this, for example when a distinction can be made between slow change and fast change. We choose to stick with this very general set of types, to show that even with a small amount of knowledge several problems can be handled.

The interaction between periods of rest and periods of change is ensured by the following rules:

$$
\begin{aligned}
& \text { terminates }(E, P) \quad \leftarrow \quad \text { init_influ }(E, I, P, S) \text {. } \\
& \text { initiates }(E, P) \quad \leftarrow \operatorname{holds\_ at}(P, E) \text {, term_influ }(E, I, P, S) \text {, } \\
& \text { not unterminated_influ }(P, E) \text {. } \\
& \text { unterminated_influ }(P, E) \leftarrow \text { init_influ }(E, I, P, \text { Sort }) \text {. } \\
& \text { unterminated_influ }(P, E) \leftarrow \text { happens }\left(E^{*}\right) \text {, influence }\left(I, E^{*}, P, S o r t\right) \text {, } \\
& \text { not term_influ }(E, I, P, \text { Sort }) \text {. }
\end{aligned}
$$

where unterminated_influ $(P, E)$ holds if there are influences on $P$ that will continue to exist after $E$.

We can now model the water tank in the following way, adding the possibility of multiple taps and plugs:

$$
\begin{array}{ll}
\text { init_influ }(E, \operatorname{tap}(Y), \text { level }(X),+)) & \leftarrow \operatorname{act}(E, \text { open_tap }(Y)) . \\
\text { init_influ }(E, \operatorname{plug}(Y), \text { level }(X),-)) & \leftarrow \operatorname{act}(E, \text { open_plug}(Y)) . \\
\text { term_influ }(E, \operatorname{tap}(Y), \text { level }(X),+)) & \leftarrow \operatorname{act}\left(E, \operatorname{close\_ tap}(Y)\right) . \\
\text { term_influ }(E, \operatorname{plug}(Y), \text { level }(X),-)) & \leftarrow \operatorname{act}(E, \text { close_plug }(Y)) .
\end{array}
$$

using the name or number of the tap to identify the influence. In this way, it is easy to determine which influence is initiated or terminated by an action.

The monotonicity, continuity and unique level constraints do not need to be modified. However, the constraints indicating that the water eventually reaches the top or the bottom - when rising or dropping - get more complicated because of the possibility of many influences: if, at a certain point in time, there are unterminated positive influences while no negative influences remain, and if after that time point there is no change of influence anymore, then the water will eventually reach its maximum level. Again a similar conclusion holds 
about the water reaching its minimum level if only negative influences exist.

$$
\begin{aligned}
& \text { false } \leftarrow \text { happens }(E) \text {, unterminated_influ }(E, \text { level }(L),+) \text {, } \\
& \text { not unterminated_influ }(E \text {, level }(L),-) \text {, } \\
& \text { not influenced_after }(E, \text { level }(L)) \text {, } \\
& \text { isa(top(l_type), l_type), not reach_after }\left(E, t o p\left(l_{-} t y p e\right)\right) \text {. } \\
& \text { false } \leftarrow \text { happens }(E) \text {, unterminated_influ }(E \text {, level }(L),-) \text {, } \\
& \text { not unterminated_influ }(E, \text { level }(L),+) \text {, } \\
& \text { not influenced_after }(E, \text { level }(L)) \text {, isa(bottom(l_type), l_type), } \\
& \text { not reach_after }(E, \text { bottom(l_type)). }
\end{aligned}
$$

with reach_after as before, and influenced_after defined as

$$
\begin{aligned}
& \text { influenced_after }(E, P) \leftarrow \text { happens }\left(E_{2}\right), E<<E_{2}, \text { term_influ }(E, I, P, S) . \\
& \text { influenced_after }(E, P) \leftarrow \text { happens }\left(E_{2}\right), E<<E_{2} \text {, init_influ }(E, I, P, S) .
\end{aligned}
$$

which completes our proposal for continuous change with multiple influences. Basically it can handle the same kinds of problems as our first proposal, but it eliminates the unrealistic restriction to one influence. An example that shows how even changes with unknown direction provide us with useful information, is the following scenario: we open a tap and a plug, resulting in an unknown change. We know nothing about the initial water level. We observe the water level at $t_{1}$ and $t_{2}$, and see it is below halfway at $t_{1}$ and above halfway at $t_{2}$. In this case, the bell should be ringing at $t_{2}$ if it is not broken. Apart from the general rules descibed above, we have

$$
\begin{aligned}
& \text { initiates }(E, \text { ring_bell }) \leftarrow \text { holds_at(level(half }), E), \text { not broken_bell } . \\
& \text { isa(bottom(l_type), l_type). } \quad \text { happens(start). } \quad \text { start }<<e_{1} \text {. } \\
& \text { isa }\left(x, l_{-t} \text { type }\right) . \quad \text { happens }\left(e_{1}\right) . \quad e_{1}<<t_{1} \text {. } \\
& \text { isa(half, l_type). happens }\left(t_{1}\right) . \quad t_{1}<<e \\
& \text { isa }(y, l \text { type }) . \quad \text { happens }(e) . \quad e<<t_{2} \text {. } \\
& \text { isa(top(l_type), l_type). happens }\left(t_{2}\right) \text {. } \\
& \operatorname{act}\left(e_{1}, \text { open_tap }\left(\operatorname{tap}_{1}\right)\right) . \quad \text { act }\left(e_{1}, \text { open_plug }\left(\text { plug } g_{1}\right)\right) \text {. }
\end{aligned}
$$

We add an extra event $e$ between $t_{1}$ and $t_{2}$. If happens were abducible, this event would always be abduced, as the constraints can never be satisfied otherwise. For the reasons indicated earlier we choose to simply add this necessary event to the scenario. Of course we do not give any information about what is going on at time $e$, it is just there to be used if needed. Similarly we add two new levels $x$ and $y$. We ask

$$
\begin{aligned}
\leftarrow & \text { not false, holds_at }\left(\text { level }(x), t_{1}\right), \text { holds_at }\left(\text { level }(y), t_{2}\right), \\
& \text { smaller }\left(l_{\_} \text {type, } x, \text { half }\right), \text { smaller }\left(l \_t y p e, \text { half }, y\right), \\
& \text { not holds_at }\left(\text { ring_bell, } t_{2}\right) .
\end{aligned}
$$

and again, we find broken_bell is abduced. Indeed, the change is not required to be monotonic, and we do not know how the water level behaves between $t_{1}$ and $t_{2}$, whether it reaches the top or the bottom, or how many times it passes the level halfway. Yet we do know, because of continuity, that it passes the level 
halfway at least once between $t_{1}$ and $t_{2}$. Therefore, if the bell is not ringing, it has to be broken.

We conclude this section by indicating why autoterminating events would lead to erroneous results in the extended version of our proposal. Suppose we use autoterminating events. If we open a tap and wait until the tank is full, such event would occur. Suppose then we do not close the tap, but open a plug. We could then conclude that there is a negative influence from the plug, but no positive one from the tap, since that influence was (auto)terminated by the event. Therefore we could conclude that the tank would empty.

However, in reality the positive influence of the tap still exists. Though it has no effect if it is the only influence present, it is not terminated and can still show itself by counteracting other influences.

This does not mean, however, that autoterminating events are a worthless notion: they can indeed occur in reality. As an example, there could be a sensor at the rim of the tank that detects the water level reaching it. This could provoke the closing of all taps. In that case, we have a real autoterminating event, and it has to be represented as such.

\section{Discussion}

We have incorporated a representation of continuous change in the Abductive Event Calculus, assuming that we have no complete knowledge about that change. We have used constraints in combination with abduction to represent the available knowledge. We have made a distinction between the influences on a changing value and the change of that value itself. This distinction is necessary if we want to model any but the most simple problem domains.

A few other authors have addressed the problem of representing continuous change in a temporal reasoning formalism. Allen's theory of time ([1]) was modified in [11] to fix certain problems arising when continuous change was considered in the original theory. Sandewall ([17], [18]) describes a framework that uses differential equations combined with logic and a form of chronological minimisation.

In an approach based like ours on the Event Calculus, Shanahan extends the formalism with trajectories([19]). These trajectories describe periods of continuous change, assuming the change is exactly known as a function of time. The extension fits in nicely with the Event Calculus, as periods of rest - described by the basic Event Calculus axioms - and periods of motion - described by the axioms for trajectories - interact without a problem. This solution is further refined in [16] to make reasoning at different levels of time granularity possible, and to allow for the parameters of the change to be modified while the change is in effect.

This approach assumes that each trajectory is exactly known. To avoid this, in [20] a qualitative version of trajectories is proposed, based on the naive physics theory of confluences described in [4]. Confluences are, simply stated, a form of qualitative differential equations. They can be used to describe the 
world in terms of the signs of certain quantities and the signs of their derivatives, without knowing any exact values. Shanahan combines these confluences with trajectories. These trajectory do not need to be exactly known anymore, but are quqlitative.

However, there are many differences between this approach and ours. Shanahan distinguishes certain landmark values through which the changing value can pass. This corresponds in a sense to our definition of levels, but the set of landmarks is always fixed while our levels and their order can be incompletely known. This results in a greater flexibility of our approach.

Another difference lies in Shanahan's treatment of autotermination using a caused event, which leads to the problems we indicated earlier. Finally, Shanahan does not distinguish influences from changes, where we argue that this distinction is necessary for handling simultaneous influences on the same variable.

Shanahan's proposal is presented in Horn clause logic, and as a logic theory provides a valid representation, but it is not intended to run as a logic program. Shanahan indicates that it still contains many loops and inefficiencies.

Our representation is compatible with the aspects of the Abductive Event Calculus that have been developed to represent indeterminism and to solve problems involving planning and diagnosis. This is probably the most important aspect of the proposal, since as far as we know all representations of continuous change to date, be it qualitative or quantitative, are only intended to solve prediction problems (if problem solving is at all possible), and can certainly not deal with indeterminism. The advantage of our proposal is that it combines the extensions for planning, diagnosis, indeterminism and continuous change in one framework.

Our theories can be - and are - actually executed by means of the SLDNFA procedure. Because of the use of many constraints and of the very high level implementation, that execution is still rather inefficient (though certain optimisations, using constraint logic programming techniques, have been included). One of our further research goals is the improvement of the abductive proof procedure, besides further representational issues.

\section{Acknowledgements}

Kristof Van Belleghem is partly supported by ESPRIT BR project Compulog II and partly by the Belgian IWONL. Marc Denecker is supported by Dienst Onderzoekscoordinatie, K.U.Leuven. Danny De Schreye is a senior research associate of the Belgian NFWO. We thank anonymous referees for valuable comments.

\section{References}

[1] J. F. Allen. Towards a General Theory of Action and Time. Artifical Intelligence, 23(11):123, 1984. 
[2] K. Clark. Negation as failure. In H. Gallaire and J. Minker, editors, Logic and databases, pages 293-322. Plenum Press, 1978.

[3] L. Console, D. Theseider Dupre, and P. Torasso. On the relationship between abduction and deduction. Journal of Logic and Computation, 1(5):661-690, 1991.

[4] J. de Kleer and J. S. Brown. A qualitative physics based on confluences. In J. Hobbs and R. Moore, editors, Formal Theories of the Commonsense World, pages 109-183. Ablex, 1985.

[5] M. Denecker. Knowledge Representation and Reasoning in Incomplete Logic Programming. $\mathrm{PhD}$ thesis, Department of Computer Science, K.U.Leuven, 1993.

[6] M. Denecker and D. De Schreye. SLDNFA; an abductive procedure for normal abductive programs. In K. Apt, editor, Proceedings of the International Joint Conference and Symposium on Logic Programming, Washington, 1992.

[7] M. Denecker, L. Missiaen, and M. Bruynooghe. Temporal reasoning with abductive event calculus. In Proceedings of ECAI 92, Vienna, 1992.

[8] K. Eshghi. Abductive planning with event calculus. In R. Kowalski and K. Bowen, editors, Proceedings of the 5th ICLP, 1988.

[9] C. Evans. The Macro-Event Calculus: Representing Temporal Granularity. In Proceedings of PRICAI, Tokyo, 1990.

[10] K. Forbus. Qualitative Process Theory. Artifical Intelligence, 24:85-168, 1984.

[11] A. Galton. A critical examination of allen's theory of action and time. Artifical Intelligence, 42:109-188, 1990.

[12] R. A. Kowalski and M. Sergot. A logic-based calculus of events. New Generation Computing, 4(4):319-340, 1986.

[13] B. Kuipers. Qualitative Simulation. Artifical Intelligence, 29:289-338, 1986.

[14] J. Lloyd and R. Topor. Making prolog more expressive. Journal of logic programming, 1(3):225-240, 1984.

[15] L. Missiaen. Localized abductive planning with the event calculus. PhD thesis, Department of Computer Science, K.U.Leuven, 1991.

[16] A. Montanari, E. Maim, E. Ciapessoni, and E. Ratto. Dealing with Time Granularity in the Event Calculus. In Proceedings of FGCS, Tokyo, pages $702-712,1992$.

[17] E. Sandewall. Combining logic and differential equations for describing real-world systems. In Proceedings 1989 Knowledge Representation Conference, page 412, 1989.

[18] E. Sandewall. Filter preferential entailment for the logic of action in almost continuous worlds. In Proceedings of IJCAI 89, page 894, 1989.

[19] M. Shanahan. Representing continuous change in the event calculus. In Proceedings of the 9th ECAI, page 598, 1990.

[20] M. Shanahan. Towards a calculus for temporal and qualitative reasoning. In Proceedings of AAAI Symposium, Stanford, 1991, 1991. 